\title{
Non-invasive prenatal testing to detect chromosome aneuploidies in 57,204 pregnancies
}

\author{
Ying Xue ${ }^{1,2+}$, Guodong Zhao ${ }^{3+}$, Hong $\mathrm{Li}^{1,2}$, Qin Zhang ${ }^{1,2}$, Jiafeng $\mathrm{Lu}^{1,2}$, Bin Yu ${ }^{4^{*}}$ and Ting Wang ${ }^{1,2^{*}}$ (D)
}

\begin{abstract}
Background: Non-invasive prenatal testing (NIPT) has been widely used to detect common fetal chromosome aneuploidies, such as trisomy 13, 18, and 21 (T13, T18, and T21), and has expanded to sex chromosome aneuploidies (SCAs) during recent years, but few studies have reported NIPT detection of rare fetal chromosome aneuploidies (RCAs). In this study, we evaluated the clinical practical performance of NIPT to analyze all 24 chromosome aneuploidies among 57,204 pregnancies in the Suzhou area of China.

Methods: This was a retrospective analysis of prospectively collected NIPT data from two next-generation sequencing (NGS) platforms (Illumina and Proton) obtained from The Affiliated Suzhou Hospital of Nanjing Medical University. NIPT results were validated by karyotyping or clinical follow-up.

Results: NIPT using the Illumina platform identified 586 positive cases; fetal karyotyping and follow-up results validated 178 T21 cases, 49 T18 cases, 4T13 cases, and 52 SCAs. On the Proton platform, 270 cases were positive during NIPT. Follow-up confirmed 85 T21 cases, 17 T18 cases, 4 T13 cases, 28 SCAs, and 1 fetal chromosome 22 aneuploidy case as true positives. There were 5 false-negative results, including 4 T21 and 1 T18 cases. The NGS platforms showed similar sensitivities and positive predictive values (PPVs) in detecting T21, T18, T13 and SCAs ( $p$ > 0.01). However, the Proton platform showed better specificity in detecting $45, X$ and the Illumina platform had better specificity in detecting T13 $(p<0.01)$. The major factor contributing to NIPT false-positives on the Illumina platform was false SCAs cases (65.11\%). Maternal chromosome aneuploidies, maternal cancers, and confined placental mosaicism caused discordant results between fetal karyotyping and NIPT.

Conclusion: NIPT with NGS showed good performance for detecting T13, T18, and T21. The Proton platform had better performance for detecting SCAs, but the NIPT accuracy rate for detecting RCAs was insufficient.
\end{abstract}

Keywords: NIPT, Chromosome aneuploidies, Next generation sequencing, Performance

\section{Introduction}

Since Lo et al. first discovered cell-free fetal DNA in the plasma of pregnant women in 1997 [1], next-generation sequencing (NGS)-based non-invasive prenatal testing (NIPT) for screening of fetal chromosome aneuploidies became reality [2]. Nowadays, NIPT has been widely used for detecting fetal chromosome trisomy 13, 18 and

\footnotetext{
* Correspondence: ybcz0519@163.com; biowt@163.com

${ }^{\dagger}$ Ying Xue and Guodong Zhao contributed equally to this work.

${ }^{4}$ Changzhou Maternity and Child Health Care Hospital affiliated to Nanjing

Medical University, Changzhou, Jiangsu Province 213003, China

'The Affiliated Suzhou Hospital of Nanjing Medical University, Suzhou

215000, Jiangsu Province, China

Full list of author information is available at the end of the article
}

21 (T13, T18, and T21) and sex chromosome aneuploidies (SCAs) with high sensitivity and specificity [3-5]. Rare fetal chromosome aneuploidies (RCAs) involve all fetal autosomal chromosomal abnormalities other than SCAs, T13, T18, and T21. However, most of the published data of NIPT have focused on three common aneuploidies (T13, T18, and T21) and SCAs [6]. The performance of NIPT for screening RCAs is still limited. Several recent reports revealed that RCAs also had great impact on prenatal diagnosis [7]. Some publications even revealed RCAs are less rare than previously thought and are often associated with poor obstetric outcomes [8]. Multiple groups have emphasized the importance of detecting RCAs for

(c) The Author(s). 2019 Open Access This article is distributed under the terms of the Creative Commons Attribution 4.0 International License (http://creativecommons.org/licenses/by/4.0/), which permits unrestricted use, distribution, and 
monitoring pregnancy health and minimizing the frequency of false-positive and false-negative NIPT results $[9,10]$.

With the development of NGS technologies, NIPT also has been applied in several sequencing platforms such as a semiconductor sequencing platform [11] and the Illumina sequencing platform [12]. With these advances, the U.S. Food \& Drug Administration and Chinese Food \& Drug Administration (CFDA) has approved NIPT to screen for common chromosomal aneuploidies. However, few studies have evaluated NIPT on different NGS platforms.

Here, we evaluated the performance of NIPT for detecting fetal T13, T18, and T21 and SCAs on Proton and Illumina sequencing platforms in pregnant women in the Suzhou area of China and examined the feasibility of using NIPT to screen for RCAs.

\section{Results}

Among 57,238 pregnancies who were undergo NIPT, 57, 204 pregnancies completed NIPT: 37,394 using the Illumina platform and 19,810 on the Proton platform, and 34 subjects were excluded due to low fetal fraction (Fig. 1). The median gestational ages at the time of blood collection were 18.0 and 17.0 weeks for the Illumina and Proton platform, respectively. The median maternal ages for pregnancies on both NGS platforms were 30.0 years. Among the pregnant women detected on Illumina platform, 22.1\% were advanced age pregnancies, $0.6 \%$ were twin pregnancies and $1.2 \%$ were IVF-ET pregnancies. While for pregnant women detected on Proton platform, 25.3\% were advanced age pregnancies, $1.1 \%$ were twin pregnancies and $2.2 \%$ were IVF-ET pregnancies (Table 1 ).

After NIPT, 586 (1.57\%) pregnancies had positive results on the Illumina platform, including 18 for T13, 71 for T18, 217 for T21, 234 for SCAs, and 46 for RCAs. Among these, 448 (76.5\%) cases underwent further prenatal diagnosis via amniocentesis; 218 fetal aneuploidies were confirmed, including 4 cases of T13, 49 cases of T18, 177 cases of T21, and 51 SCAs. For the 138 NIPTpositive cases that were not confirmed by fetal karyotyping, 114 cases refused confirmatory diagnosis, 23 cases ended with pregnancy loss, and 1 case was loss to follow-up. Among the 114 cases who decline invasive diagnostic testing, 68 cases had normal live births, three cases ended with pregnancy loss, 1 case had T21, 1 had an SCA, and 41 cases were loss to follow-up (Fig. 1).

On the Proton platform, 270 (1.36\%) pregnancies had positive NIPT results, including 23 for T13, 30 for T18, 110 for T21, 61 for SCAs, and 46 for RCAs. Among

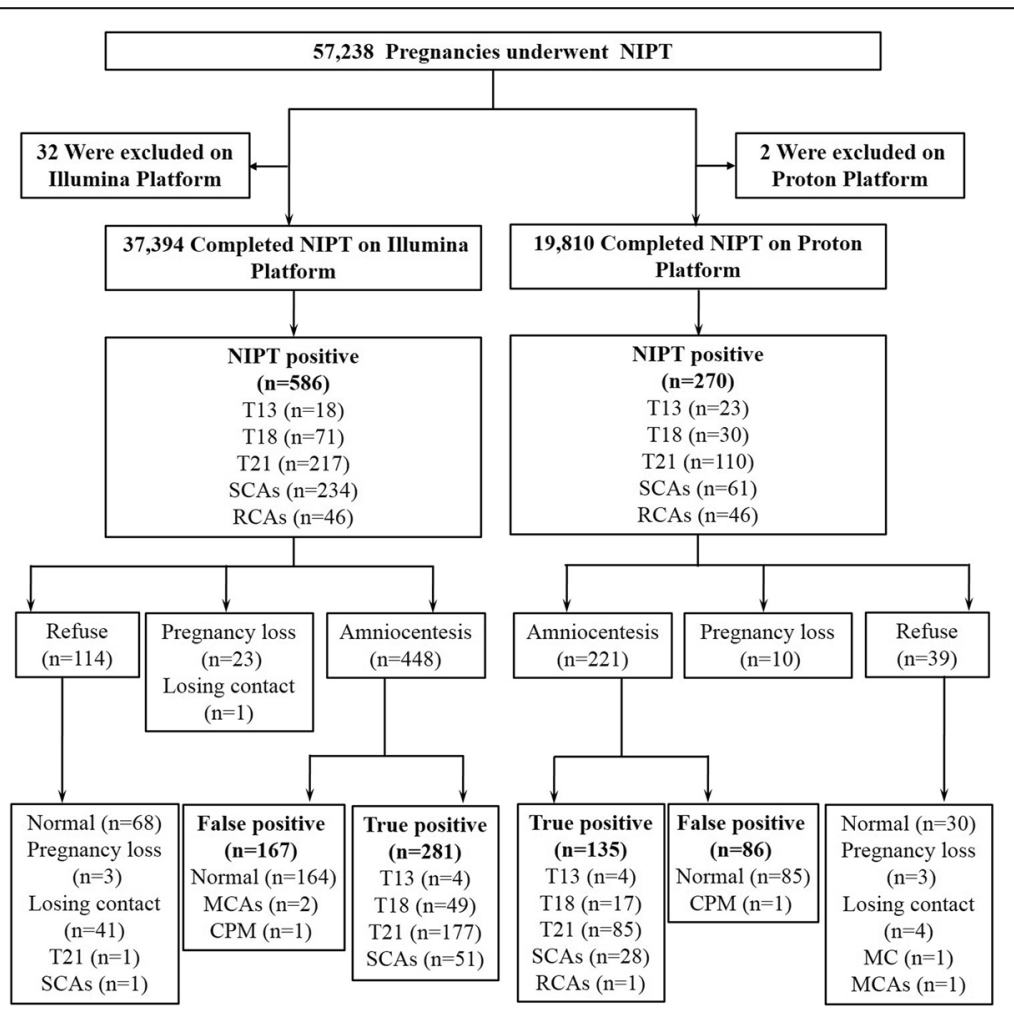

Fig. 1 Study flow chart depicting the numbers of pregnancies that used NIPT for chromosome aneuploidy screening on two NGS platforms. CPM, confined placental mosaicism; MC, maternal cancer; MCAs, maternal chromosome aneuploidies; NGS, next-generations sequencing; RCA, rare chromosomal aneuploidy; SCA, sex chromosome aneuploidy; T, trisomy 
Table 1 Distributions of maternal age and gestational age of pregnant women who underwent NIPT on two NGS platforms

\begin{tabular}{lll}
\hline & Illumina & Proton \\
\hline MA (years) & 30.0 & 30.0 \\
Median & $30.1 \pm 5.0$ & $30.4 \pm 5.0$ \\
Mean \pm SD & $16-50$ & $15-49$ \\
Min-Max & $8265(22.1 \%)$ & $5021(25.3 \%)$ \\
AA ( $\geq 35)$ & $158(1.9 \%)$ & $88(1.8 \%)$ \\
AA with positive NIPT & & \\
GA (weeks) & 18.0 & 17.0 \\
Median & $17.7 \pm 2.0$ & $17.6 \pm 2.1$ \\
$\quad$ Mean \pm SD & $11-30$ & $11-30$ \\
Min-Max & $207(0.6 \%)$ & $218(1.1 \%)$ \\
Twin pregnancies & $457(1.2 \%)$ & $429(2.2 \%)$ \\
IVF-ET pregnancies &
\end{tabular}

AA Advanced age, GA gestational age, MA maternal age, NGS

next-generation sequencing

these, 221 (81.9\%) subjects consented to amniocentesis; 135 cases were confirmed true positive including 4 cases of T13, 17 cases of T18, 85 cases of T21, 28 SCAs, and 1 RCA. Of the 49 subjects with positive NIPT results that were not confirmed by fetal karyotyping, 39 declined further testing and 10 cases ended with pregnancy loss. Among the 39 cases who refused invasive diagnostic testing, 30 had normal live births, 3 cases ended with pregnancy loss, 4 were lost to follow-up, and 2 had normal live births but their mother had chromosomal abnormality or cancer (Fig. 1).

Among the 52 true-positive SCA results on the Illumina platform, 19 were $45, \mathrm{X}, 10$ cases were $47, \mathrm{XXX}$, 17 were $47, \mathrm{XXY}$, and the remaining 6 cases were 47 , $\mathrm{XYY}$. For the 28 cases with true-positive SCA results on the Proton platform, 4 cases were $45, \mathrm{X}, 8$ were 47 , XXX, 12 were $47, \mathrm{XXY}$, and the remaining 4 were 47 , XYY. With regard to the 92 cases with positive NIPT results for RCAs (46 each for the Illumina and Proton platforms), 43 cases (18 Illumina, 25 Proton) underwent prenatal diagnosis with amniocentesis, and only 1 case was confirmed as RCA (fetal chromosome 22 aneuploidy from Proton platform, Table 2).

The sensitivities, specificities, and positive predictive values (PPVs) of NIPT using two NGS platforms for screening common chromosome aneuploidies and SCAs are shown in Table 3. Comparing performance between the two NGS platforms, there were no significant differences of sensitivity or PPV in detecting T21, T18, and $\mathrm{T} 13$, and there was no difference in specificity for detecting T21 or T18 $(p>0.01)$. However, the specificity of the Illumina platform in detecting T13 was significantly higher than that of Proton platform $(p<0.01)$.

For SCA analysis, the sensitivities of NIPT for screening each SCA type on both NGS platforms were $100.00 \%$. And the Proton platform had similar PPV in detecting SCAs compared with the Illumina platform $(p>0.01$, Table 3$)$. Regarding specificity analysis, the Proton platform showed significantly lower false positive

Table 2 NIPT results for RCAs on two NGS platforms

\begin{tabular}{|c|c|c|c|c|c|c|c|c|c|c|c|c|}
\hline \multirow[t]{2}{*}{ RCAs } & \multicolumn{4}{|c|}{ Illumina } & \multicolumn{4}{|c|}{ Proton } & \multicolumn{4}{|c|}{ Total } \\
\hline & NIP & AM & TP & PPV (\%) & $\mathrm{NIP}$ & AM & $\mathrm{TP}$ & PPV (\%) & $\mathrm{NIP}$ & AM & TP & PPV (\%) \\
\hline 7 & 15 & 5 & 0 & 0.00 & 10 & 4 & 0 & 0.00 & 25 & 9 & 0 & 0.00 \\
\hline 2 & 6 & 1 & 0 & 0.00 & 5 & 3 & 0 & 0.00 & 11 & 4 & 0 & 0.00 \\
\hline 16 & 4 & 2 & / & 0.00 & 3 & 2 & 0 & 0.00 & 7 & 4 & / & 0.00 \\
\hline 3 & 2 & 1 & 0 & 0.00 & 6 & 4 & 0 & 0.00 & 8 & 5 & 0 & 0.00 \\
\hline 22 & 1 & 1 & 0 & 0.00 & 7 & 3 & 1 & 33.33 & 8 & 4 & 1 & 25.00 \\
\hline 8 & 2 & / & / & / & 4 & 3 & 0 & 0.00 & 6 & 3 & 0 & 0.00 \\
\hline 5 & 1 & 1 & 0 & 0.00 & 3 & 2 & 0 & 0.00 & 4 & 3 & 0 & 0.00 \\
\hline 10 & 3 & 1 & 0 & 0.00 & 1 & / & / & / & 4 & 1 & 0 & 0.00 \\
\hline 14 & 3 & 2 & 0 & 0.00 & 1 & 1 & 0 & 0.00 & 4 & 3 & 0 & 0.00 \\
\hline 20 & 1 & 1 & 0 & 0.00 & 3 & 2 & 0 & 0.00 & 4 & 3 & 0 & 0.00 \\
\hline 1 & 2 & 2 & 0 & 0.00 & / & / & / & / & 2 & 2 & 0 & 0.00 \\
\hline 9 & 1 & / & / & / & 1 & / & / & I & 2 & I & I & / \\
\hline 11 & 2 & 1 & 0 & 0.00 & / & / & / & / & 2 & 1 & 0 & 0.00 \\
\hline 15 & 2 & I & / & / & I & / & / & I & 2 & I & I & / \\
\hline 19 & 1 & / & / & / & 1 & / & / & / & 2 & / & I & I \\
\hline 4 & / & / & / & / & 1 & 1 & 0 & / & 1 & / & / & / \\
\hline
\end{tabular}


Table 3 NIPT results for common fetal chromosome aneuploidies and SCAs on two NGS platforms

\begin{tabular}{|c|c|c|c|c|c|c|c|c|c|c|}
\hline \multirow{2}{*}{\multicolumn{2}{|c|}{$\begin{array}{l}\text { Chromosome } \\
\text { aneuploidies }\end{array}$}} & \multicolumn{3}{|c|}{ Illumina } & \multicolumn{3}{|l|}{ Proton } & \multicolumn{3}{|c|}{$p$-value (Illumina vs Proton) } \\
\hline & & ST (\%) & SP (\%) & PPV (\%) & ST (\%) & SP (\%) & PPV (\%) & ST & SP & PPV \\
\hline \multicolumn{2}{|l|}{21} & 98.34 & 99.94 & 88.94 & 98.84 & 99.91 & 82.52 & 0.756 & 0.169 & 0.119 \\
\hline \multicolumn{2}{|l|}{18} & 98.00 & 99.96 & 77.78 & 100.00 & 99.94 & 60.71 & 0.561 & 0.326 & 0.092 \\
\hline \multicolumn{2}{|l|}{13} & 100.00 & 99.97 & 25.00 & 100.00 & 99.91 & 18.18 & 1.000 & 0.004 & 0.611 \\
\hline \multirow[t]{4}{*}{ SCAs } & $45, x$ & 100.00 & 99.79 & 19.39 & 100.00 & 99.95 & 28.57 & 1.000 & $<0.001$ & 0.426 \\
\hline & $47, X X X$ & 100.00 & 99.98 & 55.56 & 100.00 & 99.99 & 80.00 & 1.000 & 0.330 & 0.196 \\
\hline & $47, X X Y$ & 100.00 & 99.97 & 55.17 & 100.00 & 99.99 & 92.31 & 1.000 & 0.031 & 0.018 \\
\hline & $47, X Y Y$ & 100.00 & 100.00 & 85.71 & 100.00 & 99.99 & 66.67 & 1.000 & 0.244 & 0.416 \\
\hline
\end{tabular}

NGS next-generation sequencing, PPV positive predictive value, SCA sex chromosome aneuploidies, SP specificity, ST sensitivity

rate to detect $45, \mathrm{X}$. Since most NIPT-positive RCA cases were confirmed as false positives, the PPVs for most RCAs (except fetal chromosome 22 aneuploidy) were $0 \%$ (Table 2 ).

The Illumina had 235 false-positive cases validated by fetal karyotyping and clinical follow-up, including 22 T21 cases, 16 T1 18 cases, 12 T13 cases, 153 SCAs, and 32 RCAs. Among the 118 false-positive cases identified with the Proton platform, 19 cases were T21, 12 were T18, 18 were T13, 28 were SCAs, and 39 cases were RCAs. Notably, the remaining six false-positives were due to maternal chromosome aneuploidies, confined placental mosaicism, or maternal malignancy (Table 4).

\section{Discussion}

NIPT has been widely used for detecting common fetal chromosome aneuploidies such as T13, T18, and T21 and has even expanded to SCA detection. NIPT findings about RCAs also affect prenatal diagnoses, but few studies have reported these results. We evaluated the performances of two NGS platforms for detecting all 24 chromosome aneuploidies among 57,204 pregnancies that underwent NIPT in our clinical center. In 2015, Zhang et al. reported the overall sensitivity of NIPT on Illumina platform was $99.17 \%$ for T21, $98.24 \%$ for T18, and $100 \%$ for $\mathrm{T} 13$, and the specificity was $99.95 \%$ for T21, 99.95\% for T18, and $99.96 \%$ for T13 [13]. Using the same approach, Zhou et al. showed the sensitivity and specificity of NIPT for detection of T21 and T18 and

Table 4 NIPT false-positive cases caused by maternal chromosome aneuploidies, maternal cancer, and confined placental mosaicism

\begin{tabular}{lll}
\hline NGS platforms & NIPT results & Validated results \\
\hline Illumina & $45, X$ & CPM $(45, X / 46, X Y)$ \\
& $47, X X Y$ & Maternal SCAs \\
& Chr1 aneuploidy & Maternal Chr1 aneuploidy \\
Proton & Chr7 aneuploidy & CPM (47, XX, +7/46, XX) \\
& Chr8 aneuploidy & Maternal Chr8 aneuploidy \\
& Chr22 aneuploidy & Maternal malignancy \\
\hline
\end{tabular}

T13 were 100 and 99.9\% [14]. While Francesco et al. revealed that $100 \%$ sensitivity and specificity using Proton platform for detection of T21 and T18 and T13 in a small group subjects [15]. And a system review summarized that the pooled sensitivities using different NGS platforms for T21 and T18 are 99.8\% (95\% CI 98.199.9\%) and 97.7\% (95\% CI 95.8-98.7\%) respectively, and the pooled sensitivity for $\mathrm{T} 13$ is $97.5 \%$ (95\% CI 81.9 99.7\%). The pooled specificity for all three trisomies is 99.9\% (95 99.8-99.9\%) [16]. In the present study, both Illumina and Proton platforms revealed high sensitivities and specificities for detection of T 21 and T18 and T13 (Table 3), which is comparable to previous studies. As for the PPV for detection of common fetal chromosome aneuploidies, we found similar PPVs for T21 and T18 compared to other studies, but the PPV for T13 was relatively lower in our population [14, 17]. This might due to the significantly lower incidence of T13 compared to T21 and $\mathrm{T} 18$, hence the number of T13 cases in each study was relatively low and led to PPV variation.

With regard to SCAs, we found that the Proton platform had better performance than the Illumina platform, which may be due to two reasons. Firstly, the Illumina NextSeq CN500 is based on a sequencing-by-synthesis principle, while the Proton platform uses a semiconductor sequencing technique. A previous study reported that the Illumina platform provides more accurate results than Ion Torrent Personal Genome Machine [18]. Sequencing depth and coverage are two key considerations for NGS, and greater sequencing depth and coverage always improve result accuracy [19]. In this study, the much longer reads and more uniquely mapped reads of the Proton platform narrow the gap between the two platforms, resulting in the similar sensitivities and PPVs in detecting T21, T18, T13 and SCAs, and even better specificity in detecting 45 , X. Secondly, the number of SCAs in this study was insufficient, and the sample size of Illumina platform was almost double that for the Proton platform. Despite this, the results of the two platforms for SCA detection were consistent with reports in the literature $[4,20]$. 
NIPT has been approved by the CFDA for detecting T13, T18, and T21, but it is not yet approved for SCA screening. This may be due to the high false-positive rates $(65.11 \%$ for Illumina and $24.1 \%$ for Proton) that result in relatively lower specificity and unnecessary treatment (Table 3). It is important to consider that the prevalence of SCAs is 1:500, which is more common than the major trisomies [21]. The CFDA may soon consider NIPT for SCA detection based on more clinical data and improvement of NGS methods.

For RCA analysis, chromosome 7 aneuploidy accounted for the largest proportion of NIPT-positive cases on both platforms (Table 2). According to the previous studies, T7 is the most frequently detected chromosomal abnormality $[7,10]$, which was consistent with our results. However, only one case was confirmed as a true positive among 57 , 204 cases, and the most common reason for early abortion is the existence of a major fetal chromosomal abnormality [10]. The chromosome 22 aneuploidy case was detected on the Proton platform, perhaps due to the long reads and deeper sequencing leading to an unexpected harvest, but neither NGS platform is currently optimized for detecting RCAs. The follow-up period revealed several false-positive SCAs and RCAs cases caused by confined placental mosaicism, maternal chromosome aneuploidies, and maternal cancer (Table 4). This finding is consistent with several recent studies that reported discordant results between fetal karyotyping and NIPT [22, 23].

Nevertheless, there are several limitations of our study to be considered. First, due to different sequencing principles, the plasma volume, number of uniquely mapped reads and length of reads used in this study on two NGS platforms were also different, to achieve the best performance of each platform. Which might result in some imbalance of the comparison between two NGS platforms. Hence, we could adjust the procedures of two NGS platforms in the further study, using the same initial plasma volume, for example, to make a more accurate comparison between the two NGS platforms. Secondly, the subjects used in this study had median gestational weeks 17 to 18 weeks, the early pregnancy samples in this study only occupies a small portion. Which was due to the cost of NGS based prenatal testing is relative high, it still could not be a primary screening method for prenatal diagnosis in China. In the future, with the developing of sequencing method and the reduction of sequencing costs, we believe that NIPT can be used as a primary screening method for prenatal diagnosis, and it can cover the most subjects in the early pregnancy.

\section{Conclusion}

In conclusion, both the Illumina and Proton platforms have good performance for detecting T13, T18, and T21; these NGS platforms can also be used for detecting
SCAs, but the NIPT accuracy rate for detecting RCAs remains insufficient.

\section{Materials and methods \\ Sample collection and sequencing}

This was a retrospective study based on two NGS platforms (Illumina and Proton). NIPT data were collected from Center for Reproduction and Genetics at The Affiliated Suzhou Hospital of Nanjing Medical University. The Illumina platform data were from February 1, 2012 to December 31, 2017, and Proton platform data were collected between March 1, 2015 and December 31, 2017. The study was approved by the Institutional Review Board of The Affiliated Suzhou Hospital of Nanjing Medical University. All subjects provided written informed consent prior to participation.

\section{Sequencing and data analysis on the proton platform}

Ten milliliters of peripheral blood from each pregnant woman was drawn into a $\mathrm{K}_{3}$ EDTA Vacuette tube (Becton-Dickinson, San Jose, CA, USA), and cfDNA from $600 \mu \mathrm{L}$ of maternal plasma, was captured on magnetic beads, purified and concentrated, the final cfDNA was eluted in $35 \mu \mathrm{L}$ elution buffer. And then $3 \mu \mathrm{L}$ cfDNA was used for DNA concentration measurement and the remaining $32 \mu \mathrm{L}$ cfDNA was used for the library construction following the manufacturer's protocol (Suzhou Basecare Medical Device Co., Ltd., Suzhou, Jiangsu, China), and the sequencing library was loaded onto an Ion P1 chip. A standard 500-cycle of Ion torrent sequencing was run in a single-end sequencing model [24]. All sequencing data were mapped to the human reference genome of hg19 using bowtie 2 software and four types of mapped reads (polymerase chain reaction duplicates, short reads $<35 \mathrm{bp}$, multi-mapped reads, and low-quality reads) were removed by a Perl script. The percentage of reads mapped to each chromosome was calculated using the number of uniquely mapped reads in a selected chromosome, divided by the count of uniquely mapped reads in all chromosomes (autosomal and sex) after normalizing the number of the uniquely mapping reads by LOESS regression to allow GC correction. Finally, approximately three million 125-bp uniquely mapping reads were generated, and chromosome $\mathrm{z}$ score values less than -3.0 or greater than +3.0 were classified as abnormal [24].

\section{Sequencing and data analysis on the Illumina platform}

Peripheral blood $(10 \mathrm{ml})$ from each pregnant woman was drawn into a $K_{3} E D T A$ Vacuette tube (Becton-Dickinson) or a cell-free DNA storage tube (Cwbiotech, Taizhou, Jiangsu, China). Total cfDNA was extracted from $1.2 \mathrm{~mL}$ of plasma using a nucleic acid extraction kit from Berry Genomics Co., Limited following the product 
insert protocol. DNA was captured on magnetic beads, purified through washing steps and eluted in a final volume of $42 \mu \mathrm{L}$. And then quantity of DNA extracted was assessed using Qubit 3.0 fluorometer (Invitrogen, Life technologies). DNA libraries were constructed using $40.5 \mu \mathrm{L}$ purified cfDNA following the manufacturer's protocol (Berry Genomics Co., Limited, Beijng, China). Massively parallel sequencing was performed on the Illumina NextSeq CN500 platform [23]. For each sample, approximately three million 36-bp reads were generated, of which approximately two million were uniquely mapped to the hg19 reference genome. The fetal aneuploidy status was determined by Z-scores (normal range, $-3<\mathrm{Z}<3)[25,26]$.

\section{Chromosome karyotype analysis}

Pregnant women with positive NIPT results for chromosome aneuploidies consented to invasive prenatal diagnosis. Amniocentesis was performed under sterile conditions and ultrasound guidance in our center. The amniocytes and peripheral blood cells were cultured at $37^{\circ} \mathrm{C}$. A total of 60 dividing phases were counted using an AI chromosome image analysis system based on the principle of "An International System for Human Cytogenetic Nomenclature, ISCN2013," and 20 G-banded metaphases from each sample were analyzed in triplicate [27].

\section{Data analysis}

For both Proton and Illumina sequencing platforms, a low fetal fraction of $<3 \%$ was reported as a result failure, and pregnant women with failure results after the initial blood sampling were followed up using a retest of a second blood sampling. Subjects were excluded if fetal fraction still lower than $3 \%$ at second testing.

Data were subjected to statistical analysis with IBM SPSS for Windows Version 22.0, and $t$-tests were used for comparisons between two samples at a significance level of $p<$ 0.01 . The sensitivity, specificity, and PPV of NIPT for detecting fetal chromosome aneuploidies were calculated.

\section{Abbreviations}

AA: Advanced age; AM: Amniocentesis; CFDA: Chinese Food \& Drug Administration; CPM: Confined placental mosaicism; GA: Gestational age; IVFET: In vitro fertilization and embryo transfer; MA: Maternal age; MC: Maternal cancer; MCAs: Maternal chromosome aneuploidies; NGS: Next-generation sequencing; NIP: NIPT positive; NIPT: Non-invasive prenatal testinghas; PPV: Positive predictive value; RCAs: Rare fetal chromosome aneuploidies; SCAs: Sex chromosome aneuploidies; SP: Specificity; ST: Sensitivity; T13: Trisomy 13; T18: Trisomy 13; T21: Trisomy 21; TP: True positive

\section{Acknowledgements}

Not applicable.

\section{Authors' contributions}

$Y X$ and GZ performed the statistical analyses and drafted the manuscript. HL, QZ, JL, BY and TW participated in data analysis. YX, GZ, JL, BY and TW conceived of the study and participated in the design and coordination of the study. All authors read and approved the final manuscript.

\section{Funding}

This work was supported by the Suzhou Science and Technology Support Program (SYS201649), Suzhou Key Medical Center (SZZX201505), Jiangsu Maternal and Children Health Care Research Project (F201603), Jiangsu Provincial Medical Innovation Team (CXTDB2017013), Suzhou Clinical Medical Expert Team (SZYJTD201708), and Jiangsu Maternal and Children Health Care Key Discipline (FXK201748, FXK201754).

\section{Availability of data and materials}

The data used to support the findings of this study are included within the article.

Ethics approval and consent to participate

The study was approved by the Institutional Review Board of The Affiliated Suzhou Hospital of Nanjing Medical University. All subjects provided written informed consent prior to participation.

Consent for publication

Not applicable.

\section{Competing interests}

The authors declare that they have no competing interests.

\section{Author details}

${ }^{1}$ The Affiliated Suzhou Hospital of Nanjing Medical University, Suzhou 215000, Jiangsu Province, China. ${ }^{2}$ Suzhou Municipal Hospital, Suzhou 215000, Jiangsu, China. ${ }^{3}$ Zhejiang University Kunshan Biotechnology Laboratory, Zhejiang University Kunshan Innovation Institute, Kunshan 215300, Jiangsu, China. ${ }^{4}$ Changzhou Maternity and Child Health Care Hospital affiliated to Nanjing Medical University, Changzhou, Jiangsu Province 213003, China.

Received: 11 April 2019 Accepted: 6 June 2019

Published online: 20 June 2019

References

1. Lo YD, Corbetta N, Chamberlain PF, Rai V, Sargent IL, Redman CW, et al. Presence of fetal DNA in maternal plasma and serum. Lancet. 1997; 350(9076):485-7.

2. Lau T, Cheung S, Lo P, Pursley A, Chan M, Jiang F, et al. Non-invasive prenatal testing for fetal chromosomal abnormalities by low-coverage whole-genome sequencing of maternal plasma DNA: review of 1982 consecutive cases in a single center. Ultrasound Obstet Gynecol. 2014;43(3):254-64.

3. Porreco RP, Garite TJ, Maurel K, Marusiak B, Ehrich M, van den Boom D, et al Noninvasive prenatal screening for fetal trisomies 21, 18, 13 and the common sex chromosome aneuploidies from maternal blood using massively parallel genomic sequencing of DNA. Am J Obstet Gynecol. 2014; 211(4):365.e1-e12.

4. Zhang B, Lu B-Y, Yu B, Zheng F-X, Zhou Q, Chen Y-P, et al. Noninvasive prenatal screening for fetal common sex chromosome aneuploidies from maternal blood. J Int Med Res. 2017:45(2):621-30.

5. Yu B, Lu B-Y, Zhang B, Zhang X-Q, Chen Y-P, Zhou Q, et al. Overall evaluation of the clinical value of prenatal screening for fetal-free DNA in maternal blood. Medicine. 2017;96(27):e7114.

6. Minarik G, Repiska G, Hyblova M, Nagyova E, Soltys K, Budis J, et al. Utilization of benchtop next generation sequencing platforms ion torrent PGM and MiSeq in noninvasive prenatal testing for chromosome 21 trisomy and testing of impact of in silico and physical size selection on its analytical performance. PLoS One. 2015;10(12):e0144811.

7. Pertile MD, Halks-Miller M, Flowers N, Barbacioru C, Kinnings SL, Vavrek D, et al. Rare autosomal trisomies, revealed by maternal plasma DNA sequencing, suggest increased risk of feto-placental disease. Sci Transl Med. 2017;9(405): eaan1240.

8. Fergus S, Michael B, Rhiannon S, Katie E, Maria-Elisabeth S, Andrew M. Rare autosomal trisomies; important and not so rare. Prenat Diagn. 2018;38(10): 765-71.

9. Junhui W, Ru L, Yongling Z, Xiangyi J, Qiuxia Y, Fatao L, et al. Pregnancy outcome of autosomal aneuploidies other than common trisomies detected by noninvasive prenatal testing in routine clinical practice. Prenatal diagnosis. 
10. Xie MJ, Liang ZK, He D, Xu WW, Wu YS, Yang XX, et al. Noninvasive prenatal testing of rare autosomal aneuploidies by semiconductor sequencing. Dna \& Cell Biology. 2018;37(3). https://doi.org/10.1089/dna.2017.4075.

11. Jeon YJ, Zhou Y, Li Y, Guo Q, Chen J, Quan S, et al. The feasibility study of non-invasive fetal trisomy 18 and 21 detection with semiconductor sequencing platform. PLoS One. 2014;9(10):e110240.

12. Song Y, Liu C, Qi H, Zhang Y, Bian X, Liu J. Noninvasive prenatal testing of fetal aneuploidies by massively parallel sequencing in a prospective Chinese population. Prenat Diagn. 2013;33(7):700-6.

13. Zhang $H$, Gao Y, Jiang F, Fu M, Yuan Y, Guo Y, et al. Non-invasive prenatal testing for trisomies 21, 18 and 13: clinical experience from 146958 pregnancies. Ultrasound Obstet Gynecol. 2015;45(5):530-8.

14. Qiyin Z, Ling P, Songchang C, Fang C, Rosa H, Xiaonan Y, et al. Clinical application of noninvasive prenatal testing for the detection of trisomies 21 , 18, and 13: a hospital experience. Prenat Diagn. 2015;34(11):1061-5.

15. Crea F, Forman M, Hulme R, Old RW, Ryan D, Mazey R, et al. The IONA ${ }^{\oplus}$ test: development of an automated cell-free DNA-based screening test for fetal trisomies 13, 18, and 21 that employs the ion proton semiconductor sequencing platform. Fetal Diagn Ther. 2017;42(3):218-24.

16. Iwarsson E, Jacobsson B, Dagerhamn J, Davidson T, Bernabã E, Heibert AM. Analysis of cell-free fetal DNA in maternal blood for detection of trisomy 21 18 and 13 in a general pregnant population and in a high risk population a systematic review and meta-analysis. Acta Obstet Gynecol Scand. 2017:96(1):7-18.

17. Petersen AK, Cheung SW, Smith JL, Bi W, Ward PA, Peacock S, et al. Positive predictive value estimates for cell-free noninvasive prenatal screening from data of a large referral genetic diagnostic laboratory. American Journal of Obstetrics \& Gynecology. 2017:S0002937817311870.

18. Hoecke SVD, Verhelst J, Vuylsteke M, Saelens X. Analysis of the genetic diversity of influenza a viruses using next-generation DNA sequencing. BMC Genomics. 2015;16(1):79.

19. Sims D, Sudbery I, llott NE, Heger A, Ponting CP. Sequencing depth and coverage: key considerations in genomic analyses. Nat Rev Genet. 2014; 15(2):121-32.

20. Petersen AK, Cheung SW, Smith JL, Bi W, Ward PA, Peacock S, et al. Positive predictive value estimates for cell-free noninvasive prenatal screening from data of a large referral genetic diagnostic laboratory. Am J Obstet Gynecol. 2017;217(6):13.

21. Nicolaides KH, Musci TJ, Struble CA, Syngelaki A. Gil MdM. Assessment of fetal sex chromosome aneuploidy using directed cell-free DNA analysis. Fetal Diagn Ther. 2014;35(1):1-6.

22. Amant F, Verheecke M, Wlodarska I, Dehaspe L, Brady P, Brison N, et al. Presymptomatic identification of cancers in pregnant women during noninvasive prenatal testing. JAMA Oncology. 2015;1(6):814-9.

23. Mao J, Wang T, Wang B-J, Liu Y-H, Li H, Zhang J, et al. Confined placental origin of the circulating cell free fetal DNA revealed by a discordant noninvasive prenatal test result in a trisomy 18 pregnancy. Clin Chim Acta. 2014;433:190-3.

24. Wang T, He Q, Li H, Ding J, Wen P, Zhang Q, et al. An optimized method for accurate fetal sex prediction and sex chromosome aneuploidy detection in non-invasive prenatal testing. PLoS One. 2016;11(7):e0159648.

25. Liang $\mathrm{D}, \mathrm{Lv}$ W, Wang $H$, Xu L, Liu J, Li H, et al. Non-invasive prenatal testing of fetal whole chromosome aneuploidy by massively parallel sequencing. Prenat Diagn. 2013;33(5):409-15.

26. Song Y, Huang S, Zhou X, Jiang Y, Qi Q, Bian X, et al. Non-invasive prenatal testing for fetal aneuploidies in the first trimester of pregnancy. Ultrasound Obstet Gynecol. 2015:45(1):55-60.

27. Wang T, Duan C, Shen C, Xiang J, He Q, Ding J, et al. Detection of complex deletions in chromosomes 13 and 21 in a fetus by noninvasive prenatal testing. Mol Cytogenet. 2016;9(1):3.

\section{Publisher's Note}

Springer Nature remains neutral with regard to jurisdictional claims in published maps and institutional affiliations.

Ready to submit your research? Choose BMC and benefit from:

- fast, convenient online submission

- thorough peer review by experienced researchers in your field

- rapid publication on acceptance

- support for research data, including large and complex data types

- gold Open Access which fosters wider collaboration and increased citations

- maximum visibility for your research: over $100 \mathrm{M}$ website views per year

At $\mathrm{BMC}$, research is always in progress.

Learn more biomedcentral.com/submissions 